\title{
Cell and cancer biology meld
}

\section{The startling similarity of part of epidermal growth factor and the product of a viral oncogene is another leap forward in understanding the basis of cancer. A definite link to human cancer remains elusive.}

FOR some time, there has been speculation that many viral oncogenes would turn out to be derived from cellular genes for growth factors or their receptors, so when Waterfield et al. ${ }^{1}$ and Doolittle et al. ${ }^{2}$ showed, seven months ago, that one of the two peptides that constitute plateletderived growth factor (PDGF) is similar in sequence to the transforming protein encoded by the sis oncogene of simian sarcoma virus, there was eager anticipation of the next example. That has now materialized with the paper on page 521 , in which Waterfield and his associates ${ }^{3}$ reveal that an oncogene of a tumour virus of birds is almost certainly derived from the gene for the cell surface receptor for epidermal growth factor (EGF). The result is as dramatic as it will be important for research on normal and abnormal (cancer) growth of cells. But it is not as simple as it appears at first sight.

The EGF receptor gene has been a good candidate for a viral oncogene because ${ }^{4}$ it shares with at least five viral oncogenes (of which the prototype is src of Rous sarcoma virus) the activity of a tyrosine phosphokinase, that is an enzyme which phosphorylates tyrosine residues in proteins. It has therefore been on the cards that one of the tyrosine phosphokinase encoding oncogenes would closely resemble the EGF receptor gene. If so, the argument went, the tumour virus would in effect be placing an EGF receptor in an inappropriate cell and/or at an inappropriate time. In the event, neither idea is exactly borne out.

The sequence of the erb-B oncogene of AEV-H, a strain of the avian erythroblastosis virus that causes erythroleukaemia and, less frequently, sarcoma in chickens, was published last November by Yamamoto et al. ${ }^{5}$. Unexpectedly, it contains a segment that is notably similar to the src oncogene and others with tyrosine phosphokinase activity. Nevertheless, at least three separate laboratories have since failed to demonstrate that the erb-B oncogene product has tyrosine phosphokinase activity of its own (an anomaly shared with the mos oncogene ${ }^{6}$ and, very recently, the $f m s$ oncogene ${ }^{7}$ ). But while the tyrosine phosphokinase activity of EGF receptor has been known for some years, the sequence of the receptor has awaited its purification in sufficient quantities.

Starting either with human placenta or with the human epidermoid carcinoma cell line A431, and with the vital assistance of monoclonal antibodies against the EGF receptor, Waterfield's group has now determined the amino acid sequence of 14 fragments of the receptor. These scraps of information have been sufficient for a computerized data search to align six of the peptides with segments of the protein encoded by $e r b-B$, with 74 identical amino acids out of 83 . The other eight peptides could not be aligned with erb-B.

Waterfield $e t a l$. conclude that the erb-B oncogene represents only part of the EGF receptor gene - cautiously, they raise the formal possibility that it comes not from the receptor gene itself but from a closely related gene - and deduce that the missing part encodes the EGF-binding domain of the receptor which is positioned on the external surface of cells. What the gene retains is the short segment of EGF receptor that spans the membrane and the large segment on the internal surface of the membrane which has tyrosine kinase activity.

The first puzzle is why, then, does erb-B not have demonstrable tyrosine phosphokinase activity? One possibility is that it does, but that the activity has been missed, for lack of rigorous testing. If so, the next puzzle is how it can be stimulated when the $e r b-B$ version of the EGF receptor lacks the EGF-binding domain and the normal receptor has low tyrosine phosphokinase activity in the absence of EGF. A clue to the solution to both puzzles may come from the recent demonstration by Tamura et al. ${ }^{8}$ of the activation of the tyrosine phosphokinase of insulin receptor by the proteolytic action of trypsin; perhaps the truncation of EGF receptor really does activate it.

It is even conceivable that the tyrosine phosphokinase activity, or lack of it, is not wholly relevant to the issue. Could it be that other activities of viral oncogenes/ growth factor receptors are more relevant to cancer? For example, is the recently demonstrated induction of the myc gene of lymphocytes as an early response to $\mathrm{PDGF}^{9}$ a more relevant clue or does that induction anyway depend on the tyrosine phosphokinase activity that PDGF induces in its receptor?

The next question that is likely to be answered by Waterfield and his associates is the intellectually more modest but technically formidable one of what the complete sequence of the EGF receptor looks like. The answer will be derived from the nucleotide sequence of its messenger RNA converted into cDNA. The sequencing is already well under way at Genentech under
Axel Ullrich, an author of Waterfield et al. The sequence will reveal more clearly the exact relationship between the EGF receptor and the viral $e r b-B$ gene product. Moreover, a comparison between the cDNAs of EGF receptor and those of the cellular $e r b-B$ gene $^{10}$ will make it easier to decide whether the two genes are identical.

The relevance of the discovery of Waterfield et al. for cancers, particularly human cancer, not caused by tumour viruses simply cannot be answered at this stage. It is, at least, clear that activation of the cellular $e r b-B$ gene by avian leukosis virus, a tumour virus of chickens that does not carry its own oncogene, can cause a variety of neoplasms, particularly erythroblastosis". Therefore, by analogy with $m y c$ genes $^{12,13}$, it is possible that the human cellular $e r b-B$ gene can be inappropriately activated by, for example, chromosome translocations. A thorough comparison of the expression of $e r b-B$ EGF receptor genes in healthy and tumour tissues should at least indicate how frequently they are expressed in abnormal amounts or in abnormal sizes (perhaps as a truncated protein) in tumours; the A431 cancer cell line was a good starting point for the EGF receptor purification precisely because it has at least fifty times more receptor than normal cells.

The discovery will also act as a spur to the purification and sequencing of other growth factor receptors, especially those with tyrosine phosphokinase activity - the PDGF, insulin and insulin-like growth factor-1 receptors. Perhaps it is from their genes that some other viral oncogenes have been derived. But it will be necessary to look further afield to account for them all, particularly if the recent identification ${ }^{14}$ of about one-third of the gene for actin as part of the oncogene of a tumour virus of cats is relevant to its ability to produce fibrosarcomas.

Peter Newmark

\footnotetext{
Waterfield, M.D. et al. Nature 304,35 (1983).

Doolittle, R.F. et al. Science 221, 275 (1983).

Downward, J. el al. Nature 307, 521 (1984).

Ushiro, H.\& Cohen, S. J. biol. Chem. 255, 8363 (1980)

Yanamoto, T. et al. Cell 35. 71 (1983).

Papkoff, J., Nigg, E.A. \& Hunter, T. Cell 33, 161 (1983).

Hanupe A Ciobe1, M Sherr C.J.\& Cialibert, L Proc nuin. Acad. Sci, U.S.A. 81, 85 (1984).

Tamura, S., Fujita-Yamaguchi, Y, \& Larner, J. J. biol. Chem. 258, $14749(1983)$

9. Kelly, K., Cochran, B.H., Stiles, (.1) \& 1.eder, P'. Cell 35. 603 (1983).

10. Vennstrom, B. \& Bishop, J.M. Cell 28, 135 (1982).

I. Fung, Y-K.T., lewis, W.Ci., Crittenden, 1..B. \& Kunk, H.J. Cell 33, 357 (1983).

12. Hayward, W.S., Neel, B.(i.\& Astrin, S.M. Nature 296.475 (1981).

3oberison. M. Nature 306, 733 (1983)

14. Naharoo. (i.. Rubbins. K.C. \& Reddy, E.P. Science 223, 63 (1984)
} 